\title{
Pneumothorax Caused by Aggressive Use of an Incentive Spirometer in a Patient With Emphysema
}

\author{
Jon-Emile S Kenny MD and Ware G Kuschner MD
}

\begin{abstract}
A 68-year-old man presented to the emergency department with a small pneumothorax following aggressive use of an incentive spirometer. The patient had a baseline chest radiograph consistent with emphysema. He was initially treated with oxygen in the emergency department, with resolution of his symptoms. The pneumothorax resolved spontaneously over a period of 3 days. The development of the pneumothorax was likely due to the patient's repeated forceful inspiratory maneuvers in the setting of emphysema and lung hyperinflation. Inspiratory resistive breathing can cause large negative swings in intrathoracic pressure, which may result in mechanical stress of lung tissue. This is the first report of a secondary pneumothorax associated with use of an incentive spirometer. Patients with bullous emphysema should be counseled to avoid frequent high intensity maneuvers with an incentive spirometer if the potential benefits of the procedure are marginal. Key words: incentive spirometry; pneumothorax; Müller maneuver; emphysema; transpulmonary pressure; barotrauma. [Respir Care 2013;58(7):e77-e79]
\end{abstract}

\section{Introduction}

Incentive spirometers are widely used in the postoperative setting to prevent pulmonary complications such as atelectasis, pneumonia, and respiratory failure. Incentive spirometry is designed to mimic natural sighing or yawning, by prompting the user to take long, slow, deep breaths. ${ }^{1-4}$ The maneuver decreases pleural pressure, increases transpulmonary pressure, and increases inspiratory volumes, promoting lung expansion. Incentive spirometry is widely accepted as a safe procedure, and has been studied specifically in patients who have undergone both upper and lower abdominal surgery, as well as thoracic surgery. Additionally, incentive spirometry is recommended in patients with COPD if there is concern for postoperative

The authors are affiliated with the Pulmonary Section, Veterans Affairs Palo Alto Health Care System, and with the Division of Pulmonary and Critical Care Medicine, Stanford University School of Medicine, Palo Alto, California.

The authors have disclosed no conflicts of interest.

Correspondence: Ware Kuschner MD, Pulmonary Section, Veterans Affairs Palo Alto Heath Care System, 3801 Miranda Avenue, 111P, Palo Alto CA 94304. E-mail: kuschner@stanford.edu.

DOI: $10.4187 /$ respcare. 02130 atelectasis. ${ }^{4}$ The clinical efficacy of incentive spirometry, however, remains controversial. ${ }^{1,4}$ To our knowledge, there have been no previous reports describing an association between aggressive use of an incentive spirometer and development of a pneumothorax.

\section{Case Report}

A 68-year-old man presented to the emergency department 10 days after being discharged from our medical center after undergoing an uncomplicated right total hip arthroplasty. His chief complaint was pleuritic chest pain, localized to the left hemi-thorax, which had begun abruptly. He also complained of the contemporaneous onset of shortness of breath and sweating. In the emergency department his vital signs were normal and his $\mathrm{S}_{\mathrm{pO}_{2}}$ on ambient air was $100 \%$. A chest radiograph demonstrated a left-sided pneumothorax (Figure).

The patient had a 50 pack-year history of cigarette smoking. Frontal and lateral chest radiographs obtained 2 years prior to presentation demonstrated downward displacement and flattening of the diaphragm, an increase in the retrosternal clear space, an increased antero-posterior diameter of the thorax, and attenuation of the pulmonary vasculature in the upper lung zones, consistent with hyperinflation attributable to emphysema. The remainder of his past medical and surgical history was notable for hyperlipidemia, 


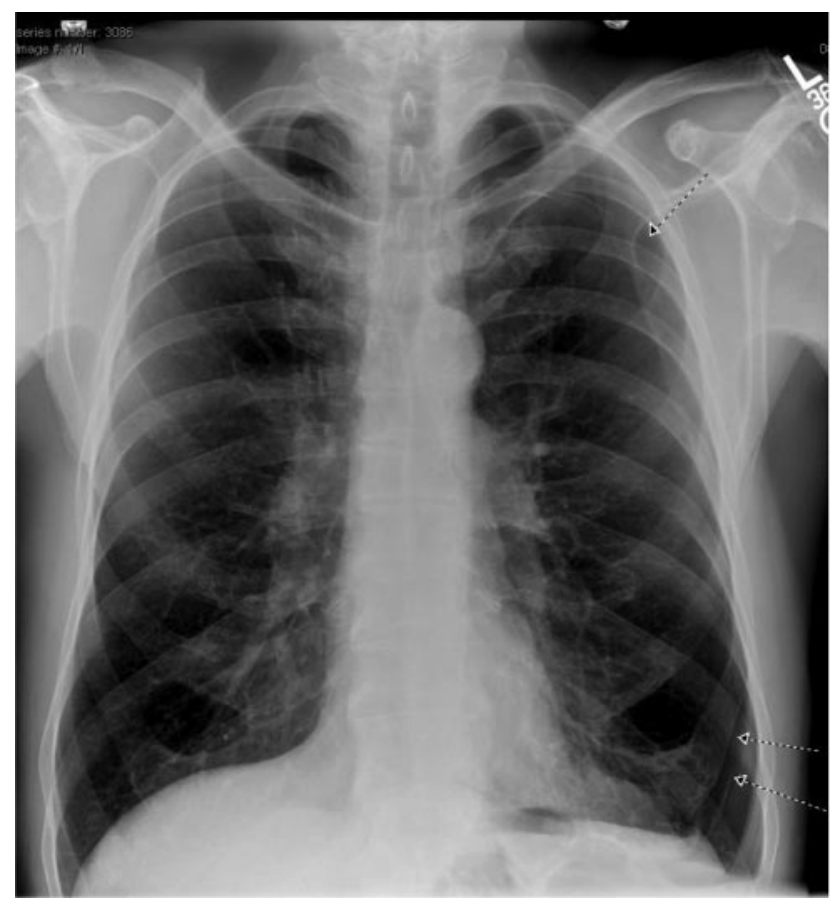

Figure. Frontal chest radiograph demonstrates a left-side pneumothorax and hyperinflation.

Barrett esophagitis, depression, insomnia, post-traumatic stress disorder, benign prostatic hyperplasia, and benign familial neutropenia. He had undergone no surgeries other than the hip replacement. His medications included multivitamins, omeprazole, tamsulosin, and aspirin. He endorsed no drug allergies and had no pertinent occupational exposures. He quit smoking 20 years prior to his presentation. His parents both died of unknown malignancies in their early 60s, and the patient has 2 sisters who are alive and without medical problems.

On further questioning, the patient described very aggressive and frequent use of his incentive spirometer (Voldyne 5000, Teleflex Medical, Research Triangle Park, North Carolina), which he had been instructed to continue to use at the time of his hospital discharge. He was discharged 36 hours postoperatively, and reported that his first use of incentive spirometry was the morning that he left our institution. He could not recall the instructions that he received from the respiratory therapist, but stated that he did not begin using the incentive spirometer regularly until the day after his discharge. He performed repeated inspiratory maneuvers, approximately $4-5$ times per hour, every hour. He characterized his inspiratory maneuvers as "very determined" and attempted to "double" the recommended 2,500 $\mathrm{mL}$ on the spirometer.

At our institution, respiratory therapists instruct patients about the indications and goals of incentive spirometry and how to use the device; however, the routine adopted by the patient was not the recommendation that he re- ceived from the respiratory therapist. He stated that his forceful approach to spirometry stemmed from his desire to "get well as quickly as possible" and that he felt that exercising his lungs repeatedly and vigorously would expedite his recovery.

The onset of his chest pain occurred 10 days post-discharge, while resting on his couch between uses of his incentive spirometer. Since leaving our institution he had not engaged in other activities that could rapidly change intrathoracic pressure (eg, heavy lifting, constipation). In the emergency department the patient was administered supplemental oxygen via non-rebreather face mask with an $\mathrm{F}_{\mathrm{IO}_{2}}$ of 1.0 for 4 hours. Oxygen was administered in an effort to promote resolution of the pneumothorax. He was discharged to home, and his pneumothorax resolved over a period of 2 days.

\section{Discussion}

This is the first report of a secondary pneumothorax associated with use of an incentive spirometer. The pneumothorax was likely due to the patient's forceful, repeated inspiratory maneuvers causing negative intrathoracic pressure deflections in the setting of underlying emphysema and lung hyperinflation. Inspiratory resistive breathing with an incentive spirometer is intended to cause strenuous contractions of the inspiratory muscles, producing large negative swings in intrathoracic pressures, similar to the Müller maneuver. The maneuver decreases pleural pressure, increases transpulmonary pressure, and results in lung expansion. This may cause stress of lung tissue, acute lung injury, and inflammation. ${ }^{5} \mathrm{~A}$ possible sequela of an abrupt increase in transpulmonary pressure is barotrauma, including pneumothorax. ${ }^{6}$

Emphysema is characterized by the destruction of lung parenchyma, leading to loss of elastic recoil, alveolar septa, and radial airway traction. This results in airway collapse, expiratory air-flow obstruction, lung hyperinflation, and gas trapping. Air spaces may enlarge and bullae may develop.

Blebs, bullae, and emphysema have been reported as potential risk factors for the development of pneumothorax in certain clinical settings. ${ }^{7-9}$ Barotrauma, including pneumothorax, pneumomediastinum, and pneumorachis attributable to repeated forceful inhalations, physiologically similar to a Müller maneuver, have been described as complications of marijuana smoking and other inhalational drug use. ${ }^{10-14}$ In our case the aggressive use of incentive spirometry, coupled with underlying emphysema and hyperinflation, was a likely explanatory factor in the development of barotrauma and the pneumothorax.

Incentive spirometry is widely used to prevent the development of postoperative atelectasis, promote the recruitment of collapsed alveoli, and reduce the risk of post- 


\section{Pneumothorax Caused by Aggressive Use of an Incentive Spirometer}

Table. Indications for Incentive Spirometry

Screening of patients who are at risk for postoperative complications by obtaining baseline flow or volume.

As a part of respiratory therapy sessions that also include deep breathing, early ambulation, and optimal analgesia to reduce the incidence of postoperative pulmonary complications.

Atelectasis or conditions that predispose to atelectasis such as:

Thoracic surgery (eg, coronary artery bypass graft)

Upper abdominal surgery

Lower abdominal surgery

Prolonged bed rest

COPD

Inadequate pain control

Abdominal or chest wall binders

Restrictive lung defects (eg, low inspiratory capacity, neuromuscular disease, spinal cord injury)

Acute chest syndrome in patients with sickle cell disease

(From reference 4.)

operative pulmonary morbidity. The intervention is generally presumed to be safe and useful, and it is recommended broadly in the postoperative period for patients at risk of developing atelectasis (Table), including patients with baseline COPD. ${ }^{4}$ There are insufficient data to recommend a specific frequency for the use of incentive spirometry. Current guidelines suggest a range from 10 maneuvers 5 times a day, to 10 breaths every hour while awake. ${ }^{4}$

While incentive spirometry is generally considered safe, there is little evidence supporting its benefit, and its routine use postoperatively is discouraged. ${ }^{4}$ This report of pneumothorax following aggressive use of incentive spirometry in a patient with emphysema and hyperinflated lungs illustrates potential harm with injudicious use of incentive spirometry. General measures of respiratory care, such as deep breathing exercises, adequate analgesia, and early ambulation, appear to be effective without the addition of incentive spirometry. ${ }^{4}$ These general practices would have been sufficient in this patient. If, however, patients with emphysema are managed with an incentive spirometer, caution is advised. This report suggests that the frequency and forcefulness of incentive spirometry maneuvers prescribed to patients should be guided by the presence of underlying emphysema or lung bullae, as well as by the underlying risk of atelectasis and pneumonia. Additionally, this report underscores the importance of counseling a patient to use an incentive spirometer as directed and to avoid extreme practices.

\section{REFERENCES}

1. Overend TJ, Anderson CM, Lucy SD, Bhatia C, Jonsson BI, Timmermans C. The effect of incentive spirometry on post-operative pulmonary complications: a systematic review. Chest 2001;120(3): 971-978.

2. Ward RJ, Danziger F, Bonica JJ, Allen GD, Bowes J. An evaluation of postoperative respiratory maneuvers. Surg Gynecol Obstet 1966; 123(1):51-54

3. Lee AB, Kinney JM, Turino G, Gump FE. Effects of abdominal operation on ventilation and gas exchange. J Natl Med Assoc 1969; 61(2):164-174.

4. Restrepo RD, Wettstein R, Wittnebel L, Tracy M; on behalf of the American Association for Respiratory Care. AARC Clinical Practice Guideline. Incentive spirometry: 2011. Respir Care 2011;56(10): 1600-1604.

5. Toumpanakis D, Kastis GA, Zacharatos P, Sigala I, Michailidou T, Kouvela $\mathrm{M}$ et al. Inspiratory resistive breathing induces acute lung injury. Am J Respir Crit Care Med 2010;182(9):1129-1136.

6. West JB. Invited Review: pulmonary capillary stress failure. J Appl Physiol 2000;89(6):2483-2489.

7. Karpman C, Aughenbaugh GL, Ryu JH. Pneumothorax and bullae in Marfan Syndrome. Respiration 2011;82(3):219-224.

8. Casali C, Stefani A, Ligabue G, Natali P, Aramini B, Torricelli P, et al. Role of blebs and bullae detected by high-resolution computer tomography and recurrent spontaneous pneumothorax. Ann Thorac Surg 2013;95(1):249-255.

9. Mohebbi I, Hassani E, Salarilak S, Bahrami AR. Do bullae and emphysema increase the risk of pneumothorax in silicosis? J Occup Med Toxicol 2007;2:8.

10. Beshay M, Kaiser H, Niedhart D, Reymond MA, Schmid RA. Emphysema and secondary pneumothorax in young adults smoking cannabis. Eur J Cardiothorac Surg 2007;32(6):834-838.

11. Hazouard E, Koninck JC, Attucci S, Fauchier-Rolland F, Brunereau L, Diot P. Pneumorachis and pneumomediastinum casued by repeated Muller's Maneuvers: complications of marijuana smoking. Ann Emerg Med 2001;38(6):694-697.

12. Luque MA, Cavallaro DL, Torres M, Emmanual P, Hillman JV. Pneumomediastinum, pneumothorax, and subcutaneous emphysema after alternate cocaine inhalation and marijuana smoking. Pediatr Emerg Care 1987;3(2):107-109.

13. Mattox KL. Pneumomediastinum in heroin and marijuana users. JACEP 1976;5(1):26-28.

14. Goodyear K, Laws D, Turner J. Bilateral spontaneous pneumothorax in a cannabis smoker. J R Soc Med 2004;97(9):435-436. 